msh-mss Mathématiques et sciences humaines

146 | Été 1999

Varia

\title{
Machiavel et la praxéologie mathématique
}

Machiavelli and mathematical praxeology

Marc Barbut

\section{OpenEdition}

Journals

Édition électronique

URL : http://journals.openedition.org/msh/2791

DOI : $10.4000 /$ msh.2791

ISSN : $1950-682$

\section{Éditeur}

Centre d'analyse et de mathématique sociales de l'EHESS

\section{Édition imprimée}

Date de publication : 1 mars 1999

ISSN : 0987-6936

\section{Référence électronique}

Marc Barbut, « Machiavel et la praxéologie mathématique », Mathématiques et sciences humaines [En ligne], 146 | Été 1999, mis en ligne le 10 février 2006, consulté le 23 juillet 2020. URL : http:// journals.openedition.org/msh/2791 ; DOI : https://doi.org/10.4000/msh.2791 
Math. Inf. Sci. hum., (37 année, $\mathrm{n}^{\circ}$ 146, 1999, pp. 19-30)

\title{
MACHIAVEL ET LA PRAXEOLOGIE MATHEMATIQUE
}

\author{
Marc BARBUT $^{1}$
}

Il y a plus de vingt-cinq ans, je publiais dans la revue Annales - Économies, Sociétés, Civilisations (25-3, mai-juin 1970) un bref article intitulé "En marge d'une lecture de Machiavel. 'L'art de la guerre' et la Praxéologie Mathématique".

A cette époque, la plupart des historiens de l'école dite des Annales n'avaient pas encore succombé aux charmes de l'anthropologie historique (ou de l'histoire anthropologique, comme on voudra), combinés avec ceux de "l'analyse des données" et des ordinateurs. Quelques-uns (je citerai notamment Robert Mandrou, Jean Meuvret et Ruggiero Romano) trouvaient quelque intérêt à une réflexion sur les rapports éventuels entre leur discipline, l'histoire de la pensée et les mathématiques.

Le colloque Mathématiques sociales et expertise qui eut lieu à l'Université de Besançon (Laboratoire de recherches philosophiques sur les logiques de l'agir) les 30 et 31 octobre 1997, a bien voulu accueillir une nouvelle version ${ }^{2}$ de mon propos, qui est de montrer combien Machiavel a été un précurseur de la formalisation mathématique ultérieure (elle commence à partir de la seconde moitié du XVII ${ }^{\text {ème }}$ siècle, et n'est pas terminée) de la logique de l'action et de la décision.

Le même exercice pourrait bien sûr être fait à propos de nombreux auteurs (K. von Clausewitz, par exemple ; mais il y en a bien d'autres) ayant médité et écrit sur ces questions. Tous ceux auxquels je pense sont largement postérieurs à Machiavel, qui me semble ainsi avoir une nette antériorité quant à la modernité de sa pensée. D'ailleurs, beaucoup de ses successeurs se sont largement nourris de lui.

Dans le texte qui suit, j'ai reproduit certains passages de mon article de 1970, mais pour l'essentiel, il est nouveau. Les idées, elles, ont peu changé.

L'édition de Machiavel qui m'a servi est celle, en français, des EEuvres complètes présentées et annotées par E. Barincou, Bibliothèque de la Pléiade, N.R.F., 1958.

Les citations, nombreuses, sont référencées par l'indication de l'ouvrage, du livre et du chapitre dont elles sont tirées. Par exemple, (G., L. V, c. 8) renvoie au Livre V, chapitre 8 de l'Art de la Guerre, (D., L. III, c. 15) au chapitre 15, Livre III du Discours sur la Première Décade.

À la fin de cet article, une bibliographie sommaire est fournie au lecteur qui souhaiterait s'initier plus avant à la Praxéologie Mathématique.

\footnotetext{
${ }^{1}$ Centre d'Analyse et de Mathématique Sociales, 54 boulevard Raspail, 75270 Paris Cedex 06, e-mail : cams@ehess.fr.

${ }^{2}$ Je remercie les organisateurs de ce colloque d'en avoir autorisé la publication ici.
} 
RÉSUMÉ - On montre comment certains des principaux concepts de la Théorie des Jeux étaient déjà clairement formulés dès le début du $16^{\text {ème }}$ siècle par Nicolas Machiavel.

MOTS-CLÉS - Jeu, duel, incertitude, moindre mal, ruse, tactique, stratégie.

SYMMARY - Machiavelli and Mathematical Praxeology

This paper is intended to point out that, as soon as the turn of $16^{\text {th }}$ century, $N$. Machiavelli clearly stated main principles of the modern Theory of Games.

KEY WORDS - Game, two persons zero sum game, uncertainty, maximin, ruse, pure strategy, mixed strategy.

Nicolas Machiavel, né et mort à Florence (1469-1527) ne fut pas seulement un écrivain et un homme de pensée.

Secrétaire, puis chef de la deuxième Chancellerie de la République de Florence, il accomplit de 1499 à 1512 plusieurs missions diplomatiques, auprès de César Borgia, Louis XII, Maximilien $1^{\text {er }}$ d'Autriche notamment.

Il s'agit d'une période particulièrement troublée et confuse de l'histoire de l'Italie, avec ses guerres, ses invasions étrangères et ses luttes entre principautés. Les succès obtenus par Machiavel dans ses missions n'en sont que plus remarquables.

Banni par les Medicis lors de leur retour au pouvoir en 1512-1513, il met ses loisirs forcés à profit pour écrire ses trois grands ouvrages de philosophie politique. C'est d'abord Le Prince, publié en 1514, puis l'Art de la Guerre, commencé à la même époque et publié en 1521 et enfin le Discours sur la Première Décade de Tite-Live écrit de 1513 à 1520, mais dont l'édition sera posthume (1532).

Cet homme, qui fut un homme d'action et, encore plus, de négociation, se met à réfléchir à l'action, à ses ressorts, à sa logique.

Et au fil de réflexions ou d'aphorismes souvent éloignés les uns des autres dans le texte, un lecteur moderne connaissant un peu la Théorie Mathématique des Jeux, voit avec étonnement et quelque ravissement, surgir et être précisément formulés certains des concepts majeurs de cette théorie.

De quoi la Théorie des Jeux (on dira encore : Praxéologie Mathématique, ou Mathématiques de la Décision) constituée au cours de ce $\mathrm{XX}^{\mathrm{ème}}$ siècle (l'ouvrage fondateur de J. von Neumann et G. Morgenstern, Theory of Games and Economic Behavior paraît en 1944) traite-t-elle en effet ? De la rationalisation des décisions que l'on doit prendre lorsque l'on est dans l'incertitude quant aux conséquences de la décision prise ; incertitude qui peut résulter soit du hasard (comme dans les loteries, les jeux de hasard, les contrats d'assurances), soit des décisions que peuvent prendre d'autres agents (d'autres joueurs) indépendants de nous (comme dans le jeu d'échecs), soit plus généralement des deux (bridge, poker).

On aura compris que pour la Théorie des Jeux (le titre même de l'ouvrage de von Neumann et Morgenstern l'indique clairement), les "jeux de société" ne sont qu'exemplaires de cas plus complexes, parce que non délimités par une "règle du jeu" précise, que l'on rencontre dans les situations considérées comme "réelles" ou "concrètes" de prise de décision, que ce soit dans la direction d'une entreprise, par exemple, ou la conduite de la guerre et des opérations militaires. 
La mathématisation de ces questions, leur modélisation mathématique, comme on dit aujourd'hui, ne s'est pas faite en un jour. Pour le cas où seul intervient le hasard, on peut la faire partir de 1654 avec Blaise Pascal (le Calcul des Probabilités) ; elle se poursuit dans les décennies qui suivent avec C. Huygens, G.W. Leibniz et surtout Jacques Bernoulli (1689, naissance de la Statistique Mathématique), se développe tout au long des siècles suivants, pour devenir de nos jours l'une des branches les plus importantes et fécondes des mathématiques.

Pour ce qui est des jeux où intervient aussi l'habileté d'autres joueurs, s'il y eut une amorce au début du XVIII ${ }^{\text {ème }}$ siècle (voir à ce sujet l'article de G. Th. Guilbaud, dans La Décision, éd. CNRS, Paris, 1961), c'est au cours du XX ${ }^{\text {ème }}$ siècle que la mathématisation s'élabore vraiment, notamment avec Émile Borel (1921), puis J. von Neumann dont les premiers travaux à ce sujet, remontent à 1928-1929, et enfin une explosion dans les décennies qui suivirent la dernière guerre mondiale, où toute l'économie théorique, mais aussi pratique (Recherche opérationnelle) en fut transformée.

Ce qu'on mathématise, quel qu'en soit le domaine, c'est toujours une théorie : ceci est particulièrement clair pour les sciences de la matière ou celles de la nature. La formalisation mathématique porte non sur l'observation du "réel", mais sur la théorie qu'en ont fait tels spécialistes du domaine étudié.

Et c'est là qu'intervient N. Machiavel ; pour qu'une Praxéologie Mathématique puisse naître, il fallait que préexiste une Praxéologie "tout court", s'exprimant discursivement.

De la science de l'action et de la décision, N. Machiavel fut, me semble-t-il, l'un des théoriciens majeurs, et l'on peut regarder son œuvre comme une pré-formalisation. On pourrait certes en dire autant d'autres ouvrages de philosophie de l'action ; ils lui sont postérieurs, et leurs auteurs se sont notamment (principalement ?) imprégnés de la lecture de Machiavel.

On a dit du Calcul des Probabilités : "c'est le bon sens mis en calcul" ; si l'on veut bien étendre cette maxime à l'ensemble de la Praxéologie Mathématique, Nicolas Machiavel fut par excellence l'homme de bon sens que nécessitait sa mise en calcul.

Parmi les textes politiques de Machiavel, ce bon sens qui se laissera "mettre en calcul", c'est dans le Discours sur la Première Décade de Tite-Live, référencé D dans la suite, lorsqu'il traite de la guerre, et l'Art de la Guerre lui-même, référencé G, que nous le trouvons surtout. Rien d'étonnant à cela : de tous les problèmes de décision dont l'analyse peut être tentée, celui de la guerre présente des éléments considérables de simplification. Il n'y a en effet, en général, que deux antagonistes, que nous désignerons par "eux" et "nous", dont les intérêts, les fins qu'ils poursuivent (le "schéma de finalité" dit le langage technique moderne) sont totalement opposés : la situation est celle d'un duel. Sur cette finalité, notre auteur est tout à fait clair: "Le but de tout gouvernement qui veut faire la guerre est de pouvoir tenir la campagne contre toute espèce d'ennemis et de vaincre le jour du combat" (G., L. I, c. 5).

Et pour vaincre, tous les moyens sont bons : "La défense de la patrie est toujours bonne, quelques moyens qu'on y emploie, ignominieux ou honorables, n'importe..." (D., L. III, c. 41). "Le point essentiel qui doit l'emporter sur tous les autres, c'est d'assurer son salut et sa liberté" (ibidem). 
La situation est nette. C'est d'ailleurs celle que l'on trouve aussi d'ordinaire dans les jeux de société à deux joueurs : tout ce qui est gagné par l'un est perdu par l'autre, et inversement. L'un et l'autre, au singulier, bien que chacun des adversaires soit le plus souvent une collectivité, "toute espèce d'ennemis", comme dans le jeu du bridge, où les deux joueurs se composent chacun de deux personnes. Ce qui fait l'unité, comme agent de décision, de chacun des adversaires collectifs, c'est précisément la communauté d'intérêts au sein d'une même collectivité : "... Les États, au contraire, où régnaient la division et le désordre voient leurs citoyens s'unir et tourner à l'avantage commun cette férocité de mœurs qui n'avait jusqu'alors enfanté que des troubles" (G., L. I, c. 11). L'unicité de l'agent de décision est d'ailleurs une nécessité de la guerre. Le chapitre 15, L. III, du Discours sur la Première Décade ne s'intitule-t-il pas "Il ne faut à une armée qu'un seul chef. Un plus grand nombre nuit" ? Et il se conclut ainsi : "... il vaut mieux mettre à la tête d'une expédition un seul chef d'une habileté ordinaire que d'en partager le commandement entre deux hommes supérieurs" (D., L. III, c. 15).

Cette unicité du commandement, il est prudent de l'organiser dans les institutions : "Une monarchie bien constituée ne donne pas à son roi une autorité sans bornes, sinon dans les armées. Là seulement, on a besoin de prendre son parti sur le champ et il ne faut pour cela qu'une seule volonté" (G., L. I, c. 4).

C'est bien la même idée que celle du général De Gaulle lorsque, hanté par le souvenir du désastre de juin 1940, il inscrira le fameux Article 16 dans la Constitution dont il dotera la France en 1958.

S'il insiste sur la nécessité pour "nous" que l'agent de décision soit unique, et qu'il y ait communauté d'intérêts, Machiavel revient, avec insistance, sur l'avantage qu'il y a inversement à diviser l'ennemi. "Un capitaine doit chercher par-dessus tout à diviser les forces qu'il a à combattre..." (G., L. VI, c. 12), "... il faut tellement les aveugler sur vos projets qu'aucun d'eux ne pense que vous êtes occupé de lui et que, négligeant de s'entraider, ils soient successivement tous écrasés, ou bien il faut leur imposer vos conditions à tous en un même jour ; chacun se croyant le seul frappé, ne songera qu'à obéir et non à résister..." (G., L. VI, c. 10).

La guerre sous sa forme la plus simple, c'est donc le duel entre deux collectivités, dont chacune a pour fin de vaincre l'autre ; restent les moyens, et c'est là-dessus que va porter tout l'effort d'analyse, aussi bien chez Machiavel qu'en Praxéologie Mathématique. Outre le "schéma de finalité", il y a le "schéma de causalité", c'est-à-dire la description exhaustive de toutes les décisions possibles, dans la situation analysée, compte tenu des moyens disponibles et des contraintes imposées, tant pour "eux" que pour "nous". Et pour chaque couple de décisions possibles, la liste et l'évaluation des conséquences que l'on en peut attendre.

Ce schéma de finalité, la Figure 1 ci-dessous, le résume (c'est ce qu'on appelle la forme normalisée du jeu); dans les jeux de société il est donné sans ambiguïté par la règle du jeu.

D'abord, donc, connaître nos propres moyens (les lignes du tableau). C'est ce que développe le chapitre 39, L. III du Discours sur la Première Décade, intitulé "Il faut qu'un capitaine connaisse les lieux où il fait la guerre". À quoi répond dans l'Art de la Guerre : "Les hommes qui méditent quelque entreprise doivent d'abord s'y disposer par tous les moyens, pour être en état d'agir à la première occasion" (G., L. I, c. 1). 


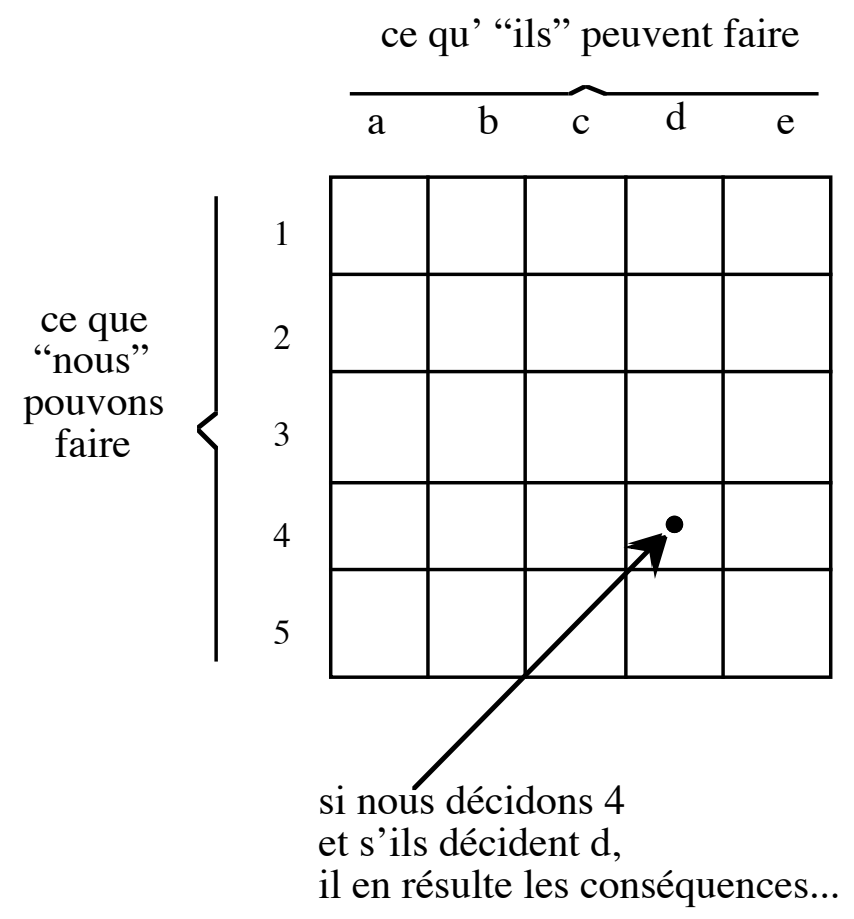

Figure 1

Ensuite, dans l'incertitude où nous sommes de ce que fera l'adversaire, prévoir tout ce qu'il peut faire (les colonnes du tableau). Qu'il faille s'efforcer de prévoir toutes les éventualités, de façon à prendre notre décision en toute connaissance de cause, cela semble évident; cependant, il y eut dans l'histoire des stratèges qui, plutôt que d'examiner tout ce que l'ennemi peut faire, ont tenté de deviner ce qu'il va faire : s'il fait autre chose, voici notre stratège devant une situation dont il n'a pas prévu les conséquences, et la défaite viendra le plus souvent sanctionner son manque de rigueur. Machiavel, lui, ne tombe pas dans le piège. Les passages abondent où il insiste sur la nécessité d'énumérer tout ce qui est possible et met en garde contre le danger de croire avoir pénétré les desseins de l'adversaire.

Certes, il intitule un chapitre du Discours sur la Première Décade (L. III, c. 18) : "Rien n'est plus digne d'un capitaine que de savoir deviner ${ }^{3}$ les desseins de l'ennemi", mais dès les premières lignes, il corrige : "... la chose la plus nécessaire et la plus utile à un commandant d'armée est de connaître les intentions et les projets de l'ennemi". Connaître, c'est tout autre chose que deviner. Voici d'ailleurs, la phrase qui suit : "Mais les connaître est chose ardue, et accroît d'autant le mérite du capitaine qui en vient à bout". Et plus loin, il y revient : "... je crois qu'un capitaine obligé de faire la guerre contre un ennemi que la nouveauté lui rend formidable, doit, s'il est sage, ..., procurer à ses soldats l'occasion de s'essayer par des escarmouches, afin qu'en apprenant peu à peu à le connaître..." (D., L. III, c. 37).

L'Art de la Guerre y insiste vigoureusement : "Or, comme un capitaine dispose toujours son armée de manière à pouvoir combattre l'ennemi qu'il voit et celui qu'il soupçonne, il faut préparer l'armée à ces deux événements..." (G., L. II, c. 7), "... ne croyez jamais que l'ennemi ne sait pas ce qu'il fait" (G., L. V, c. 7), "... l'ennemi, croyant avoir

\footnotetext{
${ }^{3}$ Les mots mis en italique dans les citations le sont par moi, M.B.
} 
deviné votre pensée, se portera à quelque mouvement que vous déjouerez aisément et vous permettra de l'écraser" (G., L. VI, c. 10). "Un capitaine doit donc parfaitement connaître les positions d'un pays, et avoir autour de lui des hommes qui en soient également instruits" (G., L. VI, c. 7) ; et ceci, qui résume tout : "... danger prévu est à demi-vaincu" (G., L. V, c. 8).

Qui a dit : "Gouverner, c'est prévoir ?" ou encore : "Un homme averti en vaut deux". Sagesse des nations...

Si une connaissance aussi complète que possible des moyens dont on dispose, de ce qui peut arriver, et une évaluation des conséquences probables des actes est, on y reviendra, nécessaire à une conduite "rationnelle", il faut cependant agir ; et l'on aborde là le troisième volet, le volet central, de la Praxéologie : les fins étant fixées, les conditions dans lesquelles se situe l'action étant analysées, des règles de conduite doivent mettre en rapport les fins et les moyens et permettre de conseiller utilement l'agent quant aux décisions à prendre parmi celles qui sont à sa disposition. Conseiller, on n'insistera jamais assez sur le mot; trop de confusions ont été faites à propos de la Théorie des Jeux : celle-ci n'est pas une théorie descriptive des comportements de l'homme agissant, mais une théorie normative, destinée à éclairer la prise de décision, à préparer l'action, et à en fournir ultérieurement des justifications aux tiers devant lesquels on serait responsable ${ }^{4}$.

À cet égard, Machiavel cite ce précepte cynique d'un auteur latin : "Je crois qu'il importe infiniment plus de délibérer sur ce qu'il faut faire que sur ce qu'il faut dire ; il sera facile, quand vous serez décidés, d'accommoder les paroles aux faits" (D., L. II, c. 15). Et il ajoute de son propre cru : "Dans l'indécision et l'incertitude de ce qu'on veut faire, il est impossible de s'expliquer ; mais, le parti une fois pris... on trouve aisément les paroles" (Ibidem).

Malgré toutes les incertitudes, quant à ce que l'adversaire va faire, et donc aux conséquences de l'action qui va être décidée, il faut agir, ici et maintenant, c'est là notre condition, dont Pascal a bien vu tout le tragique ("S'il ne fallait rien faire que pour le certain, on ne devrait rien faire..."). Que dit Machiavel ?

"Dans tous les cas, il faut toujours combattre, même avec un désavantage marqué ; car il vaut mieux tenter la fortune qui, après tout, peut être favorable, que d'attendre par irrésolution une ruine certaine" (G. L. IV, c. 5). Cette maxime dit en fait beaucoup plus que la seule nécessité d'agir, et fournit l'essentiel de la règle de conduite. Parmi les actes possibles, il y a toujours celui qui consiste à ne rien faire ; et la phrase citée ci-dessus se réfère à une situation où deux décisions sont possibles : ne rien faire, ou attaquer. Pour décider, examinez les conséquences dans chacun des deux cas; si vous attaquez vous courez, selon Machiavel, le risque d'être battu, mais vous avez une chance de gagner, d'autant plus grande que vous n'avez laissé à votre adversaire ni l'initiative ni le temps de pénétrer vos intentions et de s'y préparer, ("Notons ici qu'un prince qui veut obtenir quelque chose d'un autre, ne doit point, si l'occasion le permet, lui laisser le temps de la réflexion, mais l'acculer à une décision immédiate..." (D., L. III, c. 44)).

Si donc vous combattez, votre espérance est meilleure que si vous ne faites rien, cas où le pire, quasiment certain, car l'ennemi mettra à profit votre irrésolution, est là aussi la défaite, et où le mieux est qu'il ne se passe rien et que les choses restent en l'état.

\footnotetext{
${ }^{4}$ Vilfredo Pareto, autre italien subtil, distinguait soigneusement, lui aussi, la logique de l'action de la logique de la connaissance.
} 
La règle de conduite est donc essentiellement celle de la prudence : examiner, dans chaque cas, le pire qui puisse nous arriver, et de deux maux choisir le moindre; c'est la règle du moindre mal. C'est là le mieux, ou en tout cas le moins mauvais de ce que vous pouvez faire, en toute circonstance, et en particulier dans celles où vous êtes incapables d'estimer la probabilité que votre adversaire choisisse l'une ou l'autre des décisions qui sont à sa disposition. Cette règle du moindre mal, $\mathrm{N}$. Machiavel l'explicite admirablement au chapitre 12, L. II du Discours sur la Première Décade de Tite-Live, chapitre intitulé : "Lequel vaut mieux, lorsque l'on craint d'être attaqué, de porter la guerre chez son ennemi ou de l'attendre chez soi". Ça commence ainsi : "J'ai entendu des hommes très versés dans l'art de la guerre agiter cette question : Supposant deux princes à peu près d'égale force, si le plus puissant déclare la guerre au plus faible, est-il plus avantageux pour ce dernier d'attendre chez soi son ennemi que d'aller le chercher et de l'attaquer chez lui ? Et ils ne manquaient pas de très bonnes raisons pour et contre".

Le cadre de la décision à prendre est fixé : "Eux", qui sont un peu plus forts que "Nous", vont probablement nous faire la guerre ; c'est là l'une des décisions qui leur sont possibles, l'une des colonnes du tableau de la Figure 1 ; nous envisageons cette éventualité, et nous fixons sur elle toute notre attention. Que faire ? Nous, nous sommes devant une alternative, le tableau n'a que deux lignes : attendre, attaquer.

La Figure 2 schématise cette position du problème :

Eux

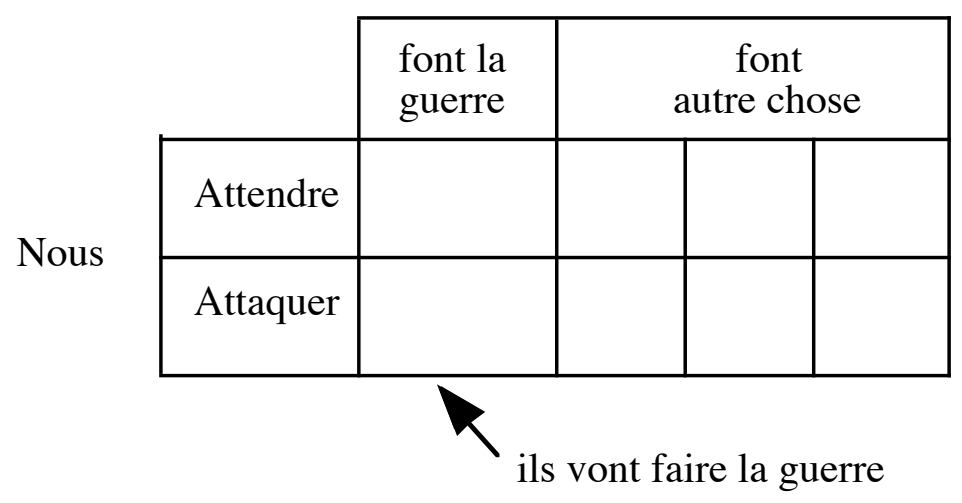

Figure 2

Comme il le fait toujours dans cet ouvrage, N. Machiavel passe alors en revue les opinions, ou les façons d'agir, de multiples personnages de l'Antiquité ou ses contemporains, voire de la mythologie. Arrivons au fait: "Mais pour dire ce que j'en pense, je crois qu'il faut faire une distinction. Ou un État est rempli de défenseurs bien armés, comme autrefois l'était celui des Romains, comme aujourd'hui celui des Suisses, ou bien il en est dépourvu, comme l'étaient autrefois les Carthaginois, et comme l'est celui de la France, ou de l'Italie" ${ }^{5}$.

Pour dire ce qu'il en pense, N. Machiavel regarde avant tout quels sont nos moyens (dont on sait qu'ils sont un peu plus faibles que ceux de l'ennemi). Ou nous sommes

\footnotetext{
5 Nous sommes, rappelons-le, au début du XVI ${ }^{\text {ème }}$ siècle ; les guerres de la France en Italie, celles de Charles VIII, Louis XII, puis François $1^{\mathrm{er}}$, sont contemporaines.
} 
"forts", ou nous sommes "faibles". Ce que je schématise, (de toute évidence, Machiavel nous y invite), par un nouveau tableau, à ne pas confondre avec les Figures 1 et 2.

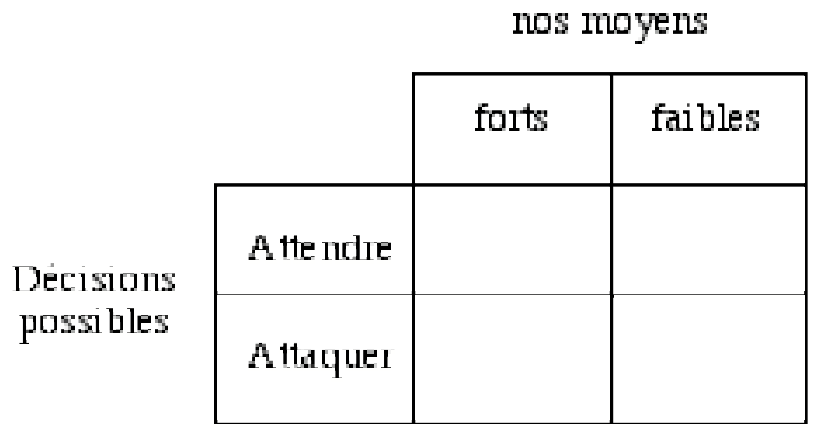

Figure 3

"Dans ce dernier cas [celui où nous sommes "faibles"], on ne saurait tenir l'ennemi trop éloigné. Toutes tes forces consistant dans tes finances et non dans tes troupes, tu es battu chaque fois que tu ne peux pas retirer cet argent par impôt ou autrement; et rien ne t'en empêche autant qu'une guerre dans tes propres foyers".

Ainsi, si nous sommes "faibles", il faut attaquer ; car si nous attendons, c'est la défaite certaine ; l'attaque laisse une chance ; c'est le moindre mal en ce cas (étant entendu que la guerre est toujours un mal). Voyons l'autre cas : "Mais quand les peuples sont armés, comme autrefois les Romains, comme aujourd'hui les Suisses, ils sont d'autant plus difficiles à vaincre qu'on les attaque de plus près. Ces états peuvent rassembler plus de forces pour repousser une invasion que pour porter la guerre chez l'ennemi".

Si donc nous sommes "forts", la prudence est d'attendre chez soi que l'ennemi attaque : nous sommes presque sûrs de vaincre ainsi, alors qu'une expédition chez l'ennemi est hasardeuse.

La Figure 4 résume cette délibération sur le parti à prendre :

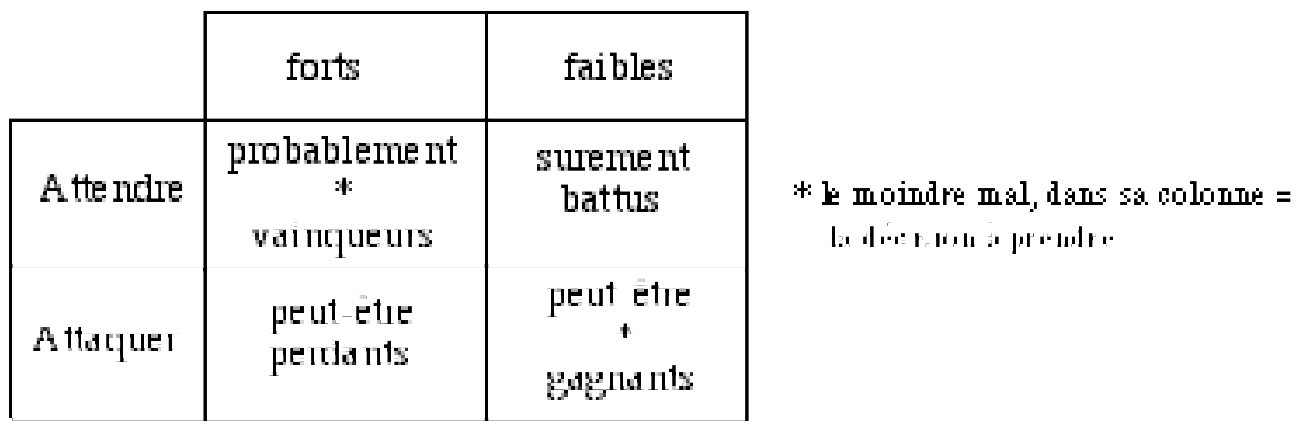

Figure 4

Ce que notre auteur exprime ainsi : "Je conclus donc de nouveau qu'un prince dont les États sont peuplés d'hommes nombreux et aguerris doit toujours attendre chez lui un ennemi puissant au lieu d'aller à sa rencontre ; mais que celui qui a des sujets désarmés et peu aguerris doit l'éloigner de son territoire le plus qu'il peut. Ainsi l'un et l'autre se défendront mieux, chacun selon ses moyens". 
Fin du chapitre 12.

La règle de conduite du moindre mal, on dit encore du maximin (maximum des minimums), nous la retrouvons en maint autre passage : "Jamais un ennemi qui vous peut vaincre par la famine ne cherchera à vous vaincre par le fer ; si la victoire, alors, n'est pas si honorable, elle est plus certaine et plus assurée" (G., L. VI, c. 8) ; "... il leur ouvrit une issue, préférant une poursuite pénible à une victoire chanceuse et durement arrachée" (G., L. VI, c. 13). Toutes ces phrases portant, d'ailleurs, la marque du réalisme de Machiavel, qui préfère la prudence au courage (comme vertu militaire); on est bien loin du "panache", mais tout près de l'éthique qui préside aux prises de décision dans les organismes économiques modernes, voire dans les états-majors : il s'agit avant tout de limiter les dégâts éventuels.

Sur le tableau à double entrée (Figure 5) qui schématise la situation générale, la règle de conduite du moindre mal se traduit par la détermination, dans chaque ligne (pour chaque acte à notre disposition) de la case dont la conséquence qui y est inscrite est la pire (dans cette ligne); puis par le choix de celle des lignes ou ce pire est le moindre.

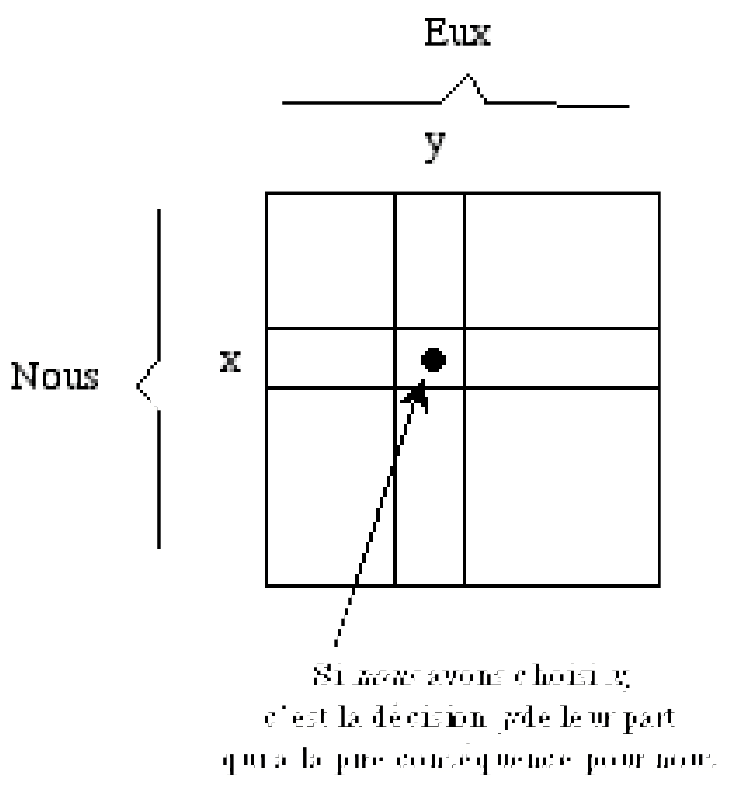

Figure 5

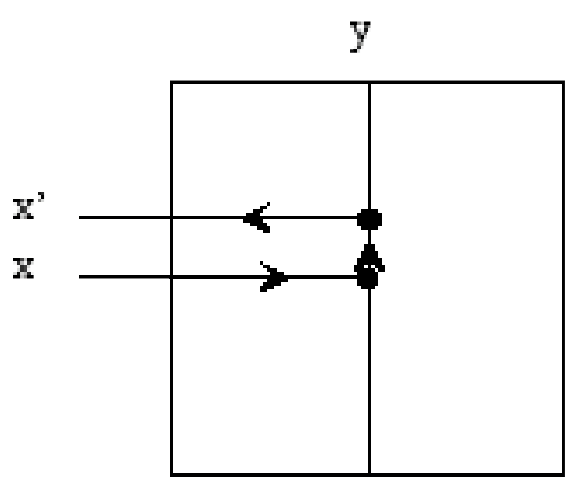

Figure 6

Mais cette règle de conduite prend une forme plus subtile lorsqu'on analyse de plus près le jeu de l'action (la nôtre) et de la réaction (la leur) dans un duel. Le danger, c'est, bien sûr, que l'ennemi devine ce que nous allons faire, soit en s'appuyant sur l'expérience qu'il a de nos décisions antérieures, qui lui apprennent à nous connaître, soit par espionnage. $\mathrm{Si}$, en effet, "ils" savent ce que nous allons faire (prendre par exemple la décision $x$ ), ils choisiront parmi les actes à leur disposition, celui, disons $y$, qui a la pire conséquence pour nous. Il faut donc feindre, ruser. Et sur ce point, Machiavel est prolixe : "La ruse sert plus que la force" (D., L. II, c. 13), "... un prince qui veut parvenir à de grandes choses doit apprendre l'art de tromper" (Ibidem). "À la guerre, la ruse mérite des éloges" (D., L. III, c. 40). "Quoique la ruse soit détestable partout ailleurs, elle est honorable à la guerre..." (Ibidem). 
Méfions-nous bien sûr des ruses de l'ennemi : "Une faute trop apparente de la part de l'ennemi doit faire soupçonner un piège" (D., L. III, c. 48). "... [il faut] se rendre compte que de telles imprudences [de l'ennemi] ne sont pas vraisemblables" (Ibidem). "Les assiégés doivent surtout se garder des pièges et des ruses de l'ennemi ; s'ils voient les assiégeants faire constamment la même chose, qu'ils entrent en défiance, et croient qu'on leur tend un piège" (G., L. VII, c. 5).

Arrêtons-nous sur ce dernier conseil. Ruser, c'est masquer ses intentions véritables ; et on doit pour cela varier ses tactiques, en changer, pour créer de l'incertitude chez l'ennemi. Nous y voici : "Dans une pareille circonstance, vous pouvez encore faire quelque mouvement qui tienne l'ennemi en suspens, soit en l'attaquant avec une partie de vos forces, de manière que, attirant de ce côté toute son attention, vous ayez le temps de sauver le reste de votre armée, soit en faisant naître quelque événement imprévu dont la nouveauté le tienne dans l'incertitude et l'embarras" (G., L. VI, c. 11). "... feindre des craintes sur quelque dessein qui ne vous donne aucune inquiétude, et dissimuler vos craintes véritables ; par-là, l'ennemi, croyant avoir pénétré votre pensée, se portera à quelque mouvement que vous déjouerez aisément et vous permettra de l'écraser" (G., L. VI, c. 10) ; "... mais ayant appris qu'Asdrubal instruit de cet ordre de bataille, voulait l'imiter, il changea au moment de la bataille toute cette disposition..." (G., L. IV, c. 1).

On ruse en bluffant, pour cacher ce qu'on va réellement faire.

Mais si d'aventure nous comprenons (ou apprenons) que l'ennemi a deviné nos intentions, nous reprenons l'avantage sur lui : nous savons maintenant qu'il a choisi $y$ (croyant que nous allions choisir $x$ ) ; nous décidons un acte, $x^{\prime}$ par exemple, qui nous donne une meilleure conséquence que $x$ lorsque l'adversaire décide $y$ (Figure 6). On voit se dessiner dans le tableau à double entrée un cheminement, résultant de la modification des décisions que prennent l'un et l'autre des antagonistes, par le jeu des devinettes : il me devine, je devine qu'il m'a deviné, il devine que j'ai devine qu'il m'a deviné, etc. Cercle vicieux qui risque d'être sans fin, et qui nous ramène à l'irrésolution dont Machiavel nous a bien dit qu'elle était la pire des conduites. Comment briser ce cercle ? Soigneusement cacher ses desseins, bien sûr, garder un secret rigoureux : "... ces dispositions sagement prises doivent être ignorées..." (G., L. I, c. 1) ; "... mais par-dessus tout, il faut que l'armée ignore à quelle expédition on la conduit : rien n'est plus utile à la guerre que de cacher ses desseins" (G., L. V, c. 8). On remarquera que pour que l'ennemi ne puisse deviner nos intentions, c'est notre propre armée, c'est-à-dire "nous", qui ne doit pas les connaître ; poussons ce principe jusqu'à sa forme ultime : "Aussi Metellus, faisant la guerre en Espagne, répondit-il à quelqu'un qui lui demandait ce qu'il ferait le lendemain : "Si ma chemise le savait, je la brûlerais sur-le-champ" (G., L. VI, c. 10). C'est quasiment la règle de conduite que von Neumann formulera bien plus tard, à peu près dans les termes suivants : si vous voulez être sûr que l'ennemi ne vous devinera pas, ignorez vous-même ce que vous allez faire ; et pour cela, ajoute von Neumann, remettez à une loterie, à un tirage au sort, à la fortune aveugle, le soin de décider pour vous.

Cette règle de conduite, qu'on pourrait appeler la "règle de Bridoison", du nom de ce juge qui, chez Rabelais, tirait ses sentences au sort, J. von Neumann démontre en effet qu'elle conduit à une généralisation de la règle du moindre mal, mais à condition de bien choisir les probabilités du tirage au sort : on peut calculer une distribution de probabilités sur l'ensemble des décisions de chacun des deux antagonistes d'un duel de sorte que chacune de ces deux distributions fournit une espérance mathématique (de l'ensemble des 
conséquences) qui est le moindre mal pour chaque joueur ; en outre, ces distributions ont les propriétés d'un "équilibre à la Cournot" : chacun des deux adversaires ne peut rien gagner et peut éventuellement perdre à modifier sa stratégie (on appelle, stratégie, ou stratégie mixte, toute distribution de probabilités sur l'ensemble des décisions possibles, ou tactiques) par rapport à celle qui correspond au moindre mal, lorsque l'autre s'y tient.

Certes, ce serait solliciter les textes que de prétendre que Machiavel a clairement vu que l'issue au cercle vicieux des "devinettes" était dans l'élargissement du champ des actes possibles de l'ensemble des tactiques, des décisions simples (les lignes du tableau à double entrée) à celui des stratégies, des mélanges de tactiques dans des proportions, avec des probabilités qui, à leur tour, se calculent en appliquant la règle du moindre mal aux espérances mathématiques qui en résultent. Mais il y a déjà chez lui l'idée du mélange pour des actes répétés dans le temps : "Il faut savoir varier suivant les temps si l'on veut toujours trouver la fortune propice" (D., L. III, c. 9).

De même, il ne me semble pas qu'il ait dégagé la notion d'équilibre, ni a fortiori celle de probabilisation de l'ensemble des décisions, surtout lorsqu'il s'agit d'un acte unique, sans répétition.

Il s'agit en effet d'une idée mathématique simple aujourd'hui, inconcevable en son temps : pour assurer l'existence d'un point fixe, et la convergence vers une limite, il faut rendre l'ensemble des décisions possibles "compact" ; et pour cela on en prend l'extension à sa "fermeture convexe".

Machiavel semble quand même avoir pressenti la solution. Les liens entre jeux de hasard pur et jeux de stratégie dans lesquels intervient l'habileté des joueurs, et la nécessité de savoir manipuler le hasard pour être un bon stratège, sont en tout cas clairement perçus : "Un capitaine de mer, habitué à combattre les vents, les flots et les hommes deviendra bien plus aisément un bon capitaine de terre, où les hommes seuls lui font tête, qu'un capitaine de terre ne deviendra un bon capitaine de mer" (G., L. I, c. 7).

Très remarquable idée, qui pourrait servir de conclusion à ces quelques observations sur ce que j'appelle la pré-formalisation, chez Machiavel, d'éléments de la Praxéologie Mathématique contemporaine.

Du point de vue adopté ici, Le Prince serait plus difficile à analyser. C'est qu'il y est question non plus seulement de la guerre (du duel), mais de situations politiques plus complexes, où interviennent plus de deux agents de décision : dès lors, les intérêts pris deux par deux ne peuvent plus être tous totalement opposés. L'étude mathématique de tels cas, commencée par Condorcet à la fin du XVIII ${ }^{\text {ème }}$ siècle, est beaucoup moins simple ; et c'est à elle que s'est consacrée, pour l'essentiel, la Théorie des Jeux dans les dernières décennies.

C'est cependant au Prince (c. 25) que j'emprunterai une conclusion, qui me semble bien résumer les liens entre la pensée de Machiavel et les mathématiques de la décision et qui fait écho à son "danger prévu est à demi vaincu" : "Je sais bien qu'aucuns furent et sont en opinion que les affaires de ce monde soient en sorte gouvernées de Dieu et de la fortune, que les hommes avec toute leur sagesse ne les puissent redresser, et n'y aient même aucune remède ; ainsi ils pourraient estimer bien vain de suer à les maîtriser, au lieu de se laisser gouverner par le sort. Cette opinion a repris du crédit en notre temps pour les grandes révolutions qu'on a vues et voit tous les jours, dépassant toute conjecture des hommes. Si bien qu'en y pensant quelques fois moi-même, en partie je me suis laissé 
tomber en cette opinion. Néanmoins, pour que notre libre arbitre ne soit éteint, j'estime qu'il peut être vrai que la fortune soit maîtresse de la moitié de nos œuvres, mais qu'etiam ${ }^{6}$ elle nous en laisse gouverner à peu près l'autre moitié".

\section{BIBLIOGRAPHIE SOMMAIRE POUR S'INITIER À LA PRAXÉOLOGIE MATHÉMATIQUE}

La meilleure initiation, pour un lecteur non mathématicien, reste à mon avis :

- GUILBAUD, G.Th., Éléments de la Théorie Mathématique des Jeux, Dunod, 1968.

Ce livre, composé d'articles publiés dans les "années 50" par la revue Économie Appliquée, est malheureusement épuisé ; les bonnes bibliothèques doivent le posséder.

Plus accessibles, semble-t-il, sont les articles "Jeux et Mathématiques", écrits par :

- ROSENSTIEHL, P., BARBUT, M., Jeux et Sports (R. Caillois, éd.), Encyclopédie de la Pléiade, Nouvelle Revue Française, 1967.

On pourra également lire avec profit :

- COUMET, E., "La théorie du hasard est-elle née du hasard ?", Annales, E.S.C., 25-3, 1970.

La décision, Colloque international, éd. CNRS, 1961.

- SAINT-SERNIN, B., Les mathématiques de la décision, Paris, Presses Universitaires de France, 1973.

On ne peut évidemment négliger l'ouvrage fondateur de la théorie moderne ; sa lecture nécessite une certaine familiarité avec les mathématiques :

- von NEUMANN, J., MORGENSTERN, O., Theory of Games and Economic Behavior, Princeton University Press, 1944.

Enfin, pour l'état actuel (années 90) de la question, telle qu'elle est enseignée aux étudiants en Économie et Économétrie, plusieurs manuels existent en langue française. Le plus récent :

- DEMANGE, G., PONSSARD, J.-P., Théorie des Jeux et Analyse économique, Paris, Presses Universitaires de France, 1994.

\footnotetext{
${ }^{6}$ En latin dans le texte.
} 